\title{
CORRIGENDUM
}

\section{Brain-immune interactions and disease susceptibility}

A Marques-Deak, G Cizza and E Sternberg

Molecular Psychiatry (2005) 10, 972. doi:10.1038/sj.mp.4001732

Correction to: Molecular Psychiatry (2005) 10, 239-250, doi:10.1038/sj.mp.4001643

Following publication of the above editorial, the author has identified the following error: In Figure
1, PNS means 'peripheral nervous system' and in lines 7 and 8 in the Figure 1 legend, the words 'and peripheral nervous system' following after 'parasympathetic nervous system' were missing. 